\title{
Proceedings of the Society of British Neurological Surgeons
}

The 96th Meeting of the Society of British Neurological Surgeons was held jointly with the Societa Italiana di Neurochirurgia in Venice 15-17 May 1980.

F IANNOTTI, HA CROCKARD, P CONFORTI, AND L SYMON (NAPLES AND LONDON) had developed an experimental model in the gerbil to study ischaemia and mechanisms of oedema formation. rCBF was evaluated by the hydrogen clearance technique and brain oedema judged by the specific gravity method. Extracranial carotid occlusion for one hour caused a $60 \%$ reduction in $\mathrm{rCBF}$ in the ipsilateral hemisphere, with a rise in brain water content. The correlation between individual postocclusive blood flow and corresponding specific gravity measurements showed that oedema developed when the focal blood flow was less than $20 \mathrm{ml} / 100 \mathrm{gm} / \mathrm{min}$, increased with progressive ischaemia and reached maximal values at flows around $7 \mathrm{ml} / 100 \mathrm{gm} /$ $\mathrm{min}$. In the no flow situation there was no oedema. To evaluate if substances like prostaglandins, released from the damaged cell membranes are involved in oedema formation, indomethacin $(3 \mathrm{mg} / \mathrm{Kg} \mathrm{b} . \mathrm{W})$ a prostaglandin synthetase inhibitor, or dexamethasone, $(2 \cdot 5 \mathrm{mg} / \mathrm{Kg} \mathrm{b}$.W), was injected intraperitoneally one hour before the carotid occlusion. Pre-treatment with either drug did not prevent oedema formation at flows of $20-12 \mathrm{ml} / 100 \mathrm{~g} / \mathrm{min}$, but considerably reduced the amount of oedema at flows around $7 \mathrm{ml} / 100 \mathrm{~g} / \mathrm{min}$. This suggests that in severe ischaemia prostaglandins or their precursors are involved in the development of oedema, while the oedema in moderate ischaemia has a different mechanism.

BA BELL, J JAKUBOWSKI, M ZAWIRSKI, N BRANSTON, D FRANCIS, A WATSON, IR PIPER, AND L SYMON (LONDON) had developed an experimental model of subarachnoid haemorrhage in the baboon to allow accurate measurements of the changes in ICP, aBP and cortical blood flows, extending over a period up to three months. Twelve hydrogen electrodes were implanted in pairs allowing CBF measurements in three standard areas of each hemisphere. Bleeding was produced by transection of posterior communicating artery with specially constructed micro- snare. The snare was implanted via a trans-orbital route. Control measurements were made in six animals and subarachnoid haemorrhage was produced in five. All animals survived and were graded clinically at 48 hours. ICP rose rapidly during the bleed, reaching $50 \mathrm{mmHg}$ in five seconds and then progressing to the average maximum mean pressure of $90 \mathrm{mmHg}$. Cerebral perfusion pressure was markedly reduced in all the animals, particularly when a cardiac arrhythmia developed during the haemorrhage. Cerebral blood flow fell dramatically in 10 minutes folling the bleed in all animals. In all grade I and II animals there was instant recovery of CBF reaching hyperaemic levels by 30 minutes. This hyperaemia was still detected in the following two days. In both grade II and IV animals flows remained low. A grade III animal had focal depression of flow in the area of cortex which corressponded to a hemiparesis, and persistent global depression of flow was found in an obtunded grade IV animal. Autoregulation was grossly impaired globally in all animals but had recovered in grade $I$ and grade II animals by five weeks. In the grade III and IV animals, autoregulation was still markedly impaired at five weeks but had returned to normal at three months.

PRR CLARKE AND J MOORE ROBERTSON (MIDDLESBROUGH) had treated 58 cases of brain abscess from a population of approximately one million between 1959 and 1979. Forty-nine were supratentorial, and nine were cerebellar. In 27 patients the middle ear was the source of infection. In 10 cases no organism had been isolated. The routine method of treatment had always been aspiration, local installation of penicillin and streptomycin, and administration of systemic antibiotics in high doses. Dexamethasone was given to 15 patients, 11 of whom made good recoveries. In the four dexamethasone cases that died, two had abscesses that had ruptured into a lateral ventricle, and undetected additional abscesses were present in the other two. Six patients had undergone craniotomy, and all had믐 survived. Forty patients had made excel- $\frac{\bar{\sigma}}{\sigma}$ ent recoveries, eight had recovered but $\overline{\bar{\omega}}$ had been left with deficits, and 10 had $\overparen{D}$ died. Fifteen patients had developed $\varrho$

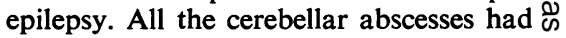
been treated by aspiration only, and had $\vec{\circ}$ survived. Three patients had died on ac- ? count of additional unsuspected abscesses $\vec{\omega}$ one because of multiple cerebral and sub- $\sigma$ dural abscesses, four because of rupture into the ventricular system, and two on account of fulminating oedema. It was felt that some of the fatal cases might have been saved had the CT scanner been? available when they were being treated. $\mathbb{H} \vec{V}$ was suggested that aspiration of the abscess together with intensive supportic therapy should remain a first line of trea $\rightarrow$ ment for cerebral abscesses and the $(D$ craniotomy should be reserved for res sistant cases.

RD HAYWARD (LONDON) had collected the : results of more conservative surgical approaches for intramedullary spinal tumours, so that new, more aggressive policies could be viewed in a realistic perspective. From the 80 cases of intra- $\frac{\circ}{\square}$ spinal glioma seen in the Department of $\varrho$ Neurosurgery, The National Hospital, $\overrightarrow{\overrightarrow{0}}$ Queen Square and at Atkinson Morleys 3 Hospital, Wimbledon, 45 cases were $\bar{T}$ selected for study. These represented the patients whose tumours involved the spinal cord alone and excluded those $\overline{\bar{J}}$ whose tumours involved the conus or 0 cauda equina since in these cases there was less controversy about the role of surgery. The picture revealed was gloomier than $\delta$ generally recognised. All the patients were 3 treated with a decompressive laminectomy, $O$ together with aspiration of any cystic fluid. Surgery to the cord itself was limited $\frac{D}{O}$ to a simple biopsy without an attempt at bulk tumour removal and even this $N$ procedure was often omitted if aspiration of a cyst provided an adequate decom- $N$ pression. Not only did this minimal N 
surgery provide no guarantee that significant deterioration would not be produced, but the average time between surgery and the moment when future deterioration (ie to paraplegia or death) occurred again was as little as 32 months. The preoperative duration of symptoms, the preoperative clinical grade and the presence or absence of a cyst had little obvious effect upon these results. Only careful comparison with the natural history of the disease as predicted by the pre-treatment and post-treatment course revealed any evidence that this mode of therapy had any clinical benefit. The reputation of gliomas of the spinal cord of a comparatively benign course following decompression and radiotherapy is generally accepted, but a review of the few series of similar size in the literature reveals that the results quoted above are by no means exceptional. It appears that the common impression is usually due to the fact that cases of ependymoma involving the conus region and cauda equina for which the prognosis is excellent) have been mixed with the spinal cord cases to give a more optimistic outlook.

SA O'LAOIRE AND DGT THOMAS (LONDON) had found surgery by the posterior approach for anteriorly placed tumour or dislocations at the upper cervical and atlanto-occipital region difficult, involving retraction of the spinal cord and medulla. The direct anterior approach, through the mouth and pharynx, provided a convenient route in operations for tumour or dislocation of the upper cervical spine. Indications for such surgery were relatively uncommon and the approach, though not new, had not been widely reported. Four transoral anterior cervical operations for spinal cord or medulla compression were described, including one for tumour of the vertebral bodies of $\mathrm{C} 2$ and $\mathrm{C} 3$, one for traumatic fracture dislocation of $\mathrm{C} 2$ and two for atlanto-axial dislocation associated with rheumatoid arthritis. One (rheumatoid) patient died of pneumonia post operatively, but all four exhibited improvement of neurological deficit following operation, and the surviving three patients made virtually full recoveries from severe neurological deficits. Healing of the pharyngeal wound did not present a problem. The transoral approach to the cervical spine presented no technical difficulties and the authors felt that the approach deserved consideration in suitable cases.
JD MILLER, SK GUDEMAN, CB WHEELER, AND LG HALLORAN (RICHMOND, VIRGINIA) had previously found in a prospective doubleblind clinical trial that cimetidine prophylaxis significantly reduced the volume of acidity of gastric contents of patients wich severe head injury. Cimetidine reduced the incidence of gastro-intestinal haemorrhage, but not of erosive mucosal lesions in the stomach, although their severity was less. They reported a new study conducted to determine whether certain clinical features of severe head injury were associated with a greater risk of gastrointestinal haemorrhage so that cimetidine prophylaxis might be more selectively employed. Total gastric secretion and gastric acidity were serially measured in 19 patients with severe head injury on days 1,3 and 6 following injury. In addition most patients underwent serial endoscopy. All had continuous ICP monitoring, were artificially ventilated and received a standard dose of steroid (dexamethasone $16 \mathrm{mg} /$ day). An assessment of GI tract haemorrhage was made in all patients. The gastric secretory response to head injury varied widely with respect to volume and titratable acidity. It did not correlate with the extent of brain shift, oculomotor dysfunction or abnormal motor responses. Gastric acidity tended, if anything, to be less in patients with very high intracranial pressure ( $>40 \mathrm{mmHg}$ ). On the whole, however, gastric $\mathrm{pH}$ was low, bleeding was common $(85 \%)$ and by the third day was associated with a high incidence of endoscopic mucosal lesions. The authors conclude that all comatose patients with head injury should receive prophylaxis for acid-related GI tract complications since neither ICP levels nor clinical signs of hemispheric or brain stem damage correlate with high gastric acidity haemorrhage or visible mucosal damage.

DJ PRICE (WAKEFIELD) reported that he had changed anticonvulsant policy from one that was rather stereotyped and traditional using phenytoin and phenobarbitone to a single drug regime some six years ago. He had chosen sodium valproate as the firstline drug and had devised a dosage schedule to achieve optimum levels in an individual patient. Of 187 patients with established epilepsy, only $43 \%$ were effectively controlled by sodium valproate with complete abolition of all seizures for a minimum of two years. A further $11 \%$ were controlled but toxicity symptoms became intolerable. Without a fully controlled cross-over trial involving a large number of patients with partial epilepsy, it was not possible to compare the effectiveness of this drug with that of others. The author gave his impression that sodium valproate compared favourably with both phenytoin and carbomezapine in these patients who tended to resist the most energetic therapy with all other drugs. For those patients with a known significant risk of developing epilepsy, it had proven highly effective. Its administration was presumed to have prevented 37 out of a further series of 143 patients from developing epilepsy in the first two years of head injury. The intravenous preparation had been used for the treatment of status epilepticus in 17 patients. Very high doses were usually necessary in the first 48 hours with subsequent reduction to an optimum maintenance dose. The drug had significant advantages over diazepam, clonazepam and paraldehyde as it had minimal side effects even at high dosage and the same drug was used for both seizure abortion and long term prophylaxis.

GFG FINDLAY AND BH CUMMINS (BRISTOL) had noticed the frequent appearance of $\bar{\sigma}$ contralateral ventricular dilatation on the CT scans of patients with a lateralisedo supratentorial tumour. 411 consecutive cases of supratentorial gliomas, meningi-ెㅡ omas or solitary metastases were reviewed excluding cases of midline tumours and those with co-existing cerebral atropy. Thirty seven per cent of the patients showed contralateral ventricular dilatation was assessed by a modification of the technique of Hahn and Rim allowing calculation of the cerebroventricular index and comparing it to age-related normal values. Contralateral ventricular dilatation was commoner with increasing age and also with increasing size of the mass. The location of the tumour had no effect on the presence of the phenomenon. Patients with a dilated ventricle had a significantly worse conscious level than those without There was no difference in the presence of hemiplegia. Compression or impingement on the foramen of Munro by the lesion was not the cause of the phenomenon. It appeared possible that it was due to occlusion or distortion of the third ventricle or aqueduct with the ipsilateral ventricle being compressed by the tumour mass. There was no evidence that the dilated contralateral ventricle acted as a balancing agent to the mass. On the 
contrary, midline shift away from the lesion was significantly commoner when the opposite ventricle was dilated. Contralateral ventricular dilatation, therefore, provides an extra intracranial burden for these patients and this is reflected in their poor conscious level.

J GARIBI, A IGARTUA AND AD ARAMENDI (BILBAO) had made a retrospective study of 201 cases of supratentorial and infratentorial meningiomas seen in a period of 20 years. As in other reports of the literature, they emphasised the predominance of women at a ratio of almost 2 to $1(64 \%)$. The peak incidence was in middle age, between 40 and 60 ; with $63 \%$ of the patients in these two decades. The extreme ages were a 4 year old girl and a 75 year old woman. The clinical features as well as the course of the disease were determined by the site of the meningioma. The main symptoms and signs were, headache $52 \%$, epilepsy $42 \%$, raised intracranial pressure $47 \%$, followed by psychological changes in $24 \%$ of the patients. One important aspect of those tumours was the duration of the disease from the first symptom to the time when the diagnosis was reached, which in their series varied from one year in $53 \%$ of the cases to more than 5 years in $11 \%$, the mean being 2,5 years. Plain skull $\mathrm{x}$-rays carried out in all the patients demonstrated bone thickening in $26 \%$ as the most frequent finding, followed by signs of raised intracranial pressure mainly in the infratentorial tumours. In the posterior fossa lesions as in other series, skull $x$-rays rarely revealed the presence and location of the tumour, but in the supratentorial cases were of great help in localisation, as was the hyperostosis in parasagittal tumours. Gamma scan was superior to the EEG, as a diagnostic aid, but of no value in the posterior fossa location. The anatomical distribution of the tumours was comparable to other series, most frequent being the parasagittal $24 \%$, convexity $18 \%$ and sphenoidal wing $15 \%$, with $11 \%$ in the posterior fossa. The pathology demonstrated a predominance of syncytial type tumours, followed by the fibroblastic $24 \%$ and with malignant type in $3 \%$. The operative mortality was $20(4 \%)$ in 196 cases. Recurrence occurred in $12 \% ; 68 \%$ of the 156 survivors did well and went back to work. The authors thought that the figures of mortality and morbidity could be reduced with early diagnosis and the newer surgical techniques.
PA STANWORTH (OXFORD) reviewed over the four year period, 1973-1977, eight babies developing hydrocephalus, who had CSF shunts inserted in the preterm period. As the survival rate of premature babies continues to increase, more cases of hydrocephalus are seen in the preterm group. These babies are very small and immature and present a new problem to the neurosurgeon. The main causes of the hydrocephalus are intraventricular and subarachnoid haemorrhage. The results were disappointing; overall mortality was $75 \%$ and that directly attributable to the surgery $63 \%$. Two out of three of the survivors were those in whom surgery was delayed. The complications were wound breakdown with CSF leak, infection and haemorrhage. It was thought that the relatively bulky shunts and the friable nature of the skin were surgical factors affecting the morbidity. It was concluded that surgery should be delayed as lcng as possible, preferably until after full term age; in the meantime, intracranial pressure should be controlled by repeated CSF taps. A small size of shunt should be used to avoid pressure necrosis and skin tension.

CGH WEST (BELFAST) described the clinical course of an 8 year old boy with posttraumatic bilateral carotid-cavernous fistulae. He noted that thirty patients with bilateral fistulae were described in the literature. Twenty one had sustained a head injury, usually severe, but in eight the fistulae appeared spontaneously. The cause in the last patient was unknown. The shunting of blood from the internal carotid artery to the cavernous sinus promotes venous hypertension and perhaps reversal of blood flow. In the presence of bilateral fistulae, neither carotid artery can be relied upon to support its fellow in maintaining cerebral perfusion, and a heavy burden is placed on the vertebrobasilar system. Compromised arterial perfusion combined with a venous hypertension encourages hemisphere ischaemia. Threat to vision, an intolerable bruit, or facial disfigurement were considered as indications for surgical intervention. The initial approach most favoured was unilateral Hunterian ligation of the common or internal carotid artery, generally followed by a trapping procedure with or without embolisation. Newer techniques of selective fistulous occlusion have been developed with the aim of preserving carotid flow. A detachable balloon catheter would seem more manoeuvrable than a muscle embolus. How well such balloons are tolerated in the long term remains to be seen. He pointed out that the diversity of methods of treatment not only reflected inherent shortcomings, but also compounded the difficulty of selecting the approach most appropriate to any particular case Central to success is a full understanding of the intracranial haemodynamics, which requires assessment of clinical and electroencephalographic tolerance of manua carotid compression, and complete angiography, including cross-compression studies.

F AFSHAR, M GRANOWSKA, CC NIMMON AND KE BRITTON (LONDON) pointed out that inert gas washout techniques for cerebral flow measurements were not suitable for studying neurological states where the distribution of pathology is likely to be asymmetrical, because assumptions about the constancy of their volume of distri $\square$ bution and the constancy and volume of partition coefficients can not be held to bo valid. They had measured independentl regional cerebral volume and regionap mean transit time using a new non invasive stochastic approach to gives regional volume flow in millilitres pefo minute. $15 \mathrm{mCi} 99 \mathrm{~m}$-Tc albumin in lesš than $0.3 \mathrm{ml}$ was administered intra을 venously using a bolus flush technique? The patient lay supine with a computer linked gamma camera placed over the vertex. A probe was set over the root of the aorta. When the bolus injection was given first pass and equilibrium data were re corded and a venous blood sample taken during the equilibrium phase. Regional volume was obtained as a fraction of total blood volume and regional mean transit time by deconvolution analysis. Thirty seven patients with subarachnoid haemorrhage were studied and classified clinically (after Hunt and Hess) into three groups and scored angiographically after Millikan as to the presence and site of arterial narrowing. These results were compared with the total and regional flows. They had demonstrated that measured total cerebral flow was significantly and somewhat better related to the clinical state of the patient than the measured degree of arterial narrowing. There was however a significant relationship between the local presence or absence of arterial narrowing and middle regional cerebral flow and the total hemisphere flow. These results suggest 
that total and regional cerebral blood flow measurements, which are made quantitatively, objectively and noninvasively using this technique, may be used to assess the state of the patient with subarachnoid haemorrhage.

RV JEFFREYS (LIVERPOOL) had made a personal prospective study on 91 consecutive cases involving direct surgery on ruptured intracranial aneurysm on the anterior part of the Circle of Willis between 1975 and 1979. The protocol involved clipping of aneurysms in all cases in pre-operative Hunt and Hess grades 1, 2 and 3 and those cases in grade 4 and 5 , in whom it was thought that hydrocephalus or intracranial haematoma was responsible for their lowly grading. During surgery, the systemic blood pressure was maintained at or near the pre-operative level. Out of 44 patients in pre-operative grades 1 and $2,93 \%$ resumed their old occupation and $7 \%$ were disabled or died. The corresponding figures for 47 patients in grades 3,4 and 5 were $60 \%$ and $40 \%$. Fifty six per cent of patients presented no post-operative problem and irrespective of their pre-operative grading, all resumed their old occupation. The outcome of 44 patients who developed early complications following surgery indicated that $55 \%$ were better than preoperative grading, when assessed at the ultimate grading of 3 months. Six patients were approximately the same and 14 worse, 12 of whom died. The aetiology and timing of early post-operative complications was discussed and it was pointed out that complications following a clipping of anterior communicating aneurysms occurred usually within the first 36 hours, whereas with internal carotid and posterior communicating aneurysms, the highest incidence of post-operative complication, in particular that due to ischaemia, came on late at 4 to 5 days post-operatively. Although hydrocephalus was present in $80 \%$ of patients at the time of surgery, only $13 \%$ showed continued long-term hydrocephalus necessitating ventricular atrial shunting.

C DI ROCCO, A IANNELLI, F LEONE, $M$ MOSCHINI, AND $\mathrm{V}$ VALORI (ROME) had diagnosed dural sinus thrombosis by clinical and angiographic findings in five patients. In four cases, the thrombotic occlusion was at the level of the superior sagittal sinus (in one, the right transverse sinus was involved as well). In the remaining patient the left cavernous sinus was occluded. Following heparin pretreatment (7500 U i.v.), urokinase (UK) was given through a continuous venous infusion (3500 U/Kg/h) for 6-9 hours; the heparinUK treatment was then continued for 2-6 days heparin, 30-40000 U/day by continuous iv infusion: $\mathrm{UK}, 3000 / \mathrm{Kg} \mathrm{U}$ iv, every 6-8 hours). Anticoagulant therapy with heparin or oral agents was administered for two months in all cases. Complete recovery occurred in all patients. The improvement was evident a few days after the beginning of treatment. Post-operative cerebral angiography performed in all but one of the patients studied, confirmed the patency of the involved sinuses in all cases. Their experience with urokinase therapy, even if confined to five cases, seemed very encouraging. (1) The favourable results obtained in all patients occurred without any major haemorrhagic complication. (2) The short period of time after drug administration and evidence of improvement in their opinion, ruled out the development of an anastomotic circulation as an explanation of the clinical recovery); (3) A direct demonstration of the thrombolytic action of urokinase was provided by by post-treatment angiographic studies, which showed the patency of the involved sinuses and canalisation of afferent cortical veins.

A AGNOLI, G MAIRA, P POLA, GF ROSSI, S RUGGIERI, L SAVI, AND A VIGNATI (L'AGUILA AND ROME) had performed extra-intracranial arterial anastomosis in 23 patients of age ranging between 16 and 68 years. The clinical picture was characterised by completed stroke (mild in 13, moderate in 8 , severe in 2 ). All the patients underwent Doppler sonography and fourvessel angiography in order to identify the location and the degree of the vascular lesion responsible for the clinical picture. The vessels affected were the internal carotid artery in 17 cases and the middle cerebral artery in 4 cases; 2 cases presented a diffuse mild atherosclerotic lesion. In order to obtain reliable criteria for surgical indication, the clinical and angiographic data were correlated with the results of CT scan and of quantitative dynamic scintigraphy (QDS). These examinations seemed to be useful to assess the presence and the degree of the cerebral lesion and to give knowledge of intracranial haemodynamics following the vascular injury.
All patients underwent surgery at least 3 weeks after the last ischaemic attack; in this way the edema phase and the risk of haemorrhagic infarction due to revascularisation was minimised. The surgical by-pass was made between a branch of the external carotid artery and a cortical branch of the middle cerebral artery. The anastomosis remained patent in 22 cases, as shown by angiography, Doppler sonography and QDS. After surgery complete neurological recovery was observed in 8 cases and a good improvement in 12 . In 3 cases the neurological deficit remained unchanged, and 1 of these died from pulmonary embolism. Some months after surgery 4 patients presented angiographic and/or clinical signs of extension of atheromatous cervical or cerebral disease, but in no case was the anastomotic site involved.

C CECOTTO, F DE NARDI AND P DEL FABRO reported nine cases of hemifacial spasm treated by posterior fossa microsurgicat decompression in the past 2 years withou砸 mortality or significant post-operative complications, and with follow up ex@ tending up to two years. In this series small vein compressing the facial nerve at the brain stem was found in 2 cases and this was coagulated and divided. In another case a small arteriovenous male formation was found, which was coagula? ted and then removed. In five cases vascular compression was caused by an artery crossing or looping the 7 th nerve: the artery was mobilised from the nerve and a piece of sponge placed between the nerve and the vessel. In one case when no apparent abnormality was found, a piece of sponge was placed around the 7 th nerve at the brain stem. Their good results and the operative findings were similar to those obtained by other authors. The relief of vascular compression in eight cases, with complete relief of hemifacial spasm in seven cases, and partial in one case, seemed to be an effective and safe treatment of this particular illness. The only case in which they did not find any apparent abnormality but which was all the same treated with a piece of sponge placed around the 7 th nerve at the brain stem, with complete relief of the spasm, seemed to confirm that the biochemical and physical modification induced by the operative microtrauma, and that a sponge on the nerve can be sufficient to modify the abnormality of function. 
A ANDREOLI, $P$ LIMONI AND $C$ TESTA (BOLOGNA) reported three cases where superficial temporal/middle cerebral anastomosis and carotid ligation had been used in the treatment of giant aneurysms. They noted that the anastomosis had application other than surgery of cerebrovascular occlusive disease; the preventive approach, eg to lesions, whose surgical treatment implies serious risks of cerebral ischaemic (carotid-cavernous sinus fistulae, benign tumours involving the base of the skull and main vessels, certain intracranial aneurysms). Althoughin aneurysm surgery the most effective approach was occlusion of the aneurysm neck, direct surgical approach to certain aneurysms might be considered particularly difficult and hazardous, and in some cases impossible. This was particularly true for "giant" aneurysms. In certain "giant" aneurysms an alternative procedure could be an indirect approach consisting of a STAMCA by-pass followed by gradual occlusion of the ipsilateral internal carotid artery by means of a Severstone clamp, which they had used in three cases. In the first case there was a right carotid ophthalmic aneurysm and a co-existing "giant" aneurysm arising from the left intracranial carotid. The right ophthalmic was clipped and later STA-MCA by-pass and carotid ligation was performed for the left aneurysm. The second case had a "giant" intracavernous saccular aneurysm which was treated with STA-MCA by-pass and carotid ligation. The third case, with a history of two previous SAH 20 and 15 years before, had a "giant" partially calcified aneurysm of the internal carotid artery. The treatment was the same as in the second case. All cases had excellent results (exclusion of the aneurysm and good collateral circulation via the patent anastomosis). Follow up 6 months/1 year.

M FRATTARELLI, C BOLLINI, P LIMONI, C GROSSI, G NUZZO, AND E POZZATI (BOLOGNA) considered 79 cases of endoventricular haemorrhage in order to evaluate prognosis. All cases were submitted to CT-scan within 48 hours of symptoms. Etiology was as follows: hypertensive haemorrhage no. $46(58 \%)$, subarachnoid haemorrhage no. $28(36 \%)$, arteriovenous malformation no. $5(6 \%)$. The patients were classified in this clinical grading: I alert or slight drowsiness, confusion and/ or cerebral cerebellar disturbance; meningeal irritation. II coma with focal cerebral or cerebellar disturbance. III sudden deep coma and primary brain-stem dysfunction. Endoventricular bleeding was classified as: complete intraventricular tamponade, partial intraventricular tamponade, intraventricular haemorrhage. The intracerebral haemotomas present in the $\mathrm{HH}$ group had the following features: large (more than 4-5 cm) no. $29(63 \%)$; small (less than 4-5 cm) no. $17(37 \%)$; basal ganglia no. 30 (66\%); white matter no. 14 $(30 \%)$; subtentorial no. $2(4 \%)$. They emphasised that the prognosis differed according to the cause of endoventricular bleeding. In $\mathbf{H H}$ the prognosis is bad (50\% mortality, $11 \%$ poor results). In their opinion the gravity of prognosis depended on clinical grading and the presence of a large haematoma. Surgical removal of 13 large haematomas ( 7 basal ganglia, 6 white matter) had favourable results only in cases of white matter haematomas. In $\mathbf{S A H}$, cases were subdivided into those who died in the acute phase and those who survived the acute phase to exclude arterial late spasm. The prognosis was even worse ( $64 \%$ mortality in the acute phase), and depended on degree of endoventricular bleeding and on clinical grading. In AVMs the prognosis was favourable, despite constant remarkable endoventricular bleeding as reported by other authors.

F FRANK, G GAIST, F SERVADEI, AND G FRANK (BOLOGNA) analysed the usefulness of CT scan control in the stereotactic determination of lesions, as a means of determining the exact lesion site, as control on possible post-operative complications, and as a means of determining the type of lesion induced. Post-operative CT scan controls were performed on 12 patients, all of whom were suffering from diseases of the extrapyramidal system. The targetpoints were the rear section of the Zona Incerta (8 patients), the VOP nucleus of the ventro-basal complex of the thalamus ( 3 patients) and the VOA nucleus (1 patient). The clinical results were obtained by evaluation a month after operation and a year after operation. In two patients, symptoms recurred a month after the lesion and in another two there was partial recurrence after a year, while in eight patients the clinical result appeared unchanged a year after the operation. From the post-operative CT scans analysis, three types of lesions could be identified: edema, necrosis and haemorrhage. From the relationship between the lesion caused stereotactically and the clinical result obtained, it appeared that: (1) short term recurrence corresponded to widespread hypodensity of the thalamus (edema). $\tau$ (2) partial recurrence after some time $\stackrel{(S)}{C}^{-}$ corresponded to irregular, hypodense lesions or haemorrhagic-necrotic lesions which were, however, always small in size, less than $3 \mathrm{~mm}$ (at CT scan control). (3) an unchanged clinical result over a long period of time corresponded to a CT scan $\bar{\tau}$ image which was increasingly larger than $3 \mathrm{~mm}$ and quite distinct in its haemorrhage or necrotic aspect. They concluded that prognosis could be made on the basis of $\triangle$ some aspects of the stereotactic lesions determined at CT scan control.

F TOMASELLO, A SPADARO AND V ALBANESE (NAPLES) reported endothelial changes in experimental models of microneurovascular anastomoses. Although the patency rate of the extra-intracraniat? bypass was high, thrombogenic and $\vec{V}$ endothelial changes following end-to-side anastomoses had not been fully investig 얌 ted. Scanning electron microscopy (SEMP was found useful for observation of the endothelial surface. The endothelial reg sponse was evaluated in experimenta models of end-to-side anastomoses haemos. dynamically reproducing those performe in clinical conditions. The left common $\infty$ carotid artery was sutured end-to-side to the contralateral one in rats. Before blood flow restoration, the brachiocephalic trunk was occluded below its bifurcation, allowing bidirectional flow towards the recipient vessel. The SEM observations were carried out at varying intervals of time, 15 and 45 minutes, 3 and 24 hours, 3, 5, 9 and 14 days following the surgical procedure. A fibrin-platelet reaction, covering the suture line and the stitches, was found in all specimens at 15 and 45 minutes. This thrombus formation gradually decreased at 3 and 24 hours. Early signs of re-endothelisation were seen at 1-3 days and were more evident at 5-9 days. The endothelial repair occurred first on the suture line and then on the stitches; it was completed at 14 days. Some conclusions could be drawn on the basis of these findings; the first hour following blood flow restoration was the most critical time for thrombus formation. Re-endothelisation occurred by advancing cells from the pre-existing endothelial layer covering the defect and the suture 
material. Lastly, the influence of some minor problems of microsurgical technique on re-endothelisation was outline. Folding of the edges of the vascular wall, intraluminal knots and large holes produced by passing the needle were causes of delay in endothelial repair.

F COLOMBO, A ZANARDO, F ANGRILLI, $\checkmark$ PINNA, AND A BENEDETTI (VICENZA AND PADUA) illustrated four cases of stereotactic thalamic lesions and their evolution studied on computer tomograms. Brain specimens of one of these lesions were analysed. The shape and size of their images wer? correlated with the probe temperature, power input of applied radiofrequency current and its duration. The CT appearance of the thalamic lesion seemed to consist of two different features. (1) A discrete central or slightly eccentric core of increased attenuation, corresponding to blood or necrotic tissue. (2) An area of decreased attenuation surrounding the central core, indicating the existence of brain oedema. In all CT slices localisation of the lesion in relation to the ventricular landmarks showed good agreement with the intended placement. This led the authors to propose a new method to obtain the stereotactic target co-ordinates directly from the CT scanner. This aim was attained by introducing a new co-ordinate system, identified by three reference points, recognisable on CT scan and on stereotactic x-rays. Early trials were performed with metallic spheres, one millimeter in diameter in radiotranslucent screws fixed to the cranial vault and left under the skin. The target was localised on CT slices in relation to ventricular landmarks. The CT scanner visualisation unit gave the reference points and target co-ordinates on CT scan co-ordinates. The three reference points were also identified on stereotactic $\mathrm{x}$-rays; their stereotactic co-ordinates were calculated by a geometrical analytical approach taking into account radiological distortion. An adequately programmed computer linked the reference points and the chosen target and calculated its coordinates on the co-ordinates system of the stereotactic device. Preliminary results obtained by this method on head phantoms were shown. A completely automatic system for stereotactic surgery foreseen, operation being possibly performed by a stereotactic motorised apparatus driven by a computer.
A BENEDETTI, C CARBONIN AND F COLOMBO (VICENZA) reported their experience of the surgical treatment of spastic syndromes at spinal level. They had performed 20 lumbar myelotomies, 19 posterior selective lumbar rhizotomies, 5 posterior cervical rhizotomies, 4 complete posterior lumbar rhizotomies and 2 anterior lumbar rhizotomies. Follow up ranged from one to four and a half years. Lumbar myelotomy was performed in patients affected by a serious spastic syndrome with impairment of motor and sensory functions, unbearable muscle spasms, and absent sphincter control. The underlying disease was multiple sclerosis in 11 and spinal cord trauma in 9 patients. Clinical results were good in 13 patients, fair in 3 . The spastic syndrome recurred in 4 . Selective posterior lumbar rhizotomy was reserved for patients with a medium degree of lower limb spasticity, and moderate impairment of voluntary motor function. Most of them were children affected by cerebral palsy. Clinical results were good in 16, fair in 1. Two patients experienced a late recurrence of spasticity. In 5 patients affected by cerebral palsy a complete posterior cervical rhizotomy was carried out. These patients complained before surgery of spastic tratraparesis with trunk and limb dyskinesias. The results were good in 3 and fair in 2. Anterior lumbar rhizotomy and complete lumbar rhizotomy were employed in those patients in which the spastic syndrome recurred after lumbar myelotomy and posterior selective lumbar rhizotomy respectively. They believed that surgical treatment of spasticity at spinal level was the last resort after any pharmacological treatment and a complate rehabilitation programme. Nevertheless, this surgery, selected with careful criteria, could afford good and long lasting results, without serious complications or mortality. These operations were carried out with the aid of operating microscope; small radicular and medullary vessels must be spared to avoid undue extension of ischaemic damage to the cord.

R GIUFFRE, N DI LORENZO AND A FORTUNA (ROME) analysed the 565 primary spinal tumours operated on at the Institute of Neurosurgery, Rome University Medical School, up to December 1979, and found 61 in children up to the age of 15 years (16 sympathetic tumours, 11 gliomasependymomas, 10 benign bone tumours, 9 sarcomas, 7 dysembryogenetic tumours, 4 neurinomas, 4 arachnoid cysts). $60 \%$ were extradural and $40 \%$ intradural tumours. Distribution over the spinal column was uniform. The first three years of life accounted for $34 \%$. Motor deficits $(86 \%)$ pain $(63 \%)$ and sphincter disturbances $(19 \%)$ were the most prominent complaints. Plain x-rays were abnormal in $65 \%$ of the patients, in a few cases permitting the identification of tumour type. CSF protein was recorded in 50 patients and was abnormal in 42.56 patients had myelography which was diagnostic in 55. CT scan was performed in two patients and in both was diagnostic. 61 patients had 63 neurosurgical interventions, the operative mortality being $1.6 \%$. Ten patients required further surgery, retroperitoneal or mediastinal. 31 patients received radiotherapy and 10 of these chemotherapy as well. Initial misdiagnosis and later referral to the neurosurgeon was common. On 15 patients of this series presented minor neurological signs when first seen. The long-term follow up (mean 8.6 years) in 43 cases shows that $77 \%$ of the patients led a useful life. A less pessi mistic attitude to the surgical treatment of spinal tumours in childhood seemsD justified. Microsurgical techniques permi attempts at total removal even in intra medullary astrocytomas. To keep down the risk of vertebral deformities after treatment, it is important to limit laminec tomy laterally and to insist on the wearing of an orthopaedic jacket for the first few months after operation.

A Pietrangeli, L Gessini, B JANDOLO, L BOVE AND C LANDUCCI (ROME) studied injuries affecting the median nerve, due to entrapment. They believed that entrapments at the pronator teres level wera quite frequent, even more so than cases reported in the literature might suggest. Mistakes in diagnosis and treatment were frequent and could result in severe disability. Clinical features of pronator teres syndrome were (1) sparing of pronator teres (the branch to it emerges from the median nerve before penetrating between the two extremes of the muscle (2) impairment of all other muscles innervated by median nerve (3) subjective and/or objective sensory troubles in cutaneous territories supplied by median nerve. EMG was standardised by testing the pronator teres, the flexor carpi radialis and the thenar eminence muscles; the evaluation of distal latency (motor and 
sensory) and nerve conduction velocity of the median nerve on elbow-wrist and axilla-elbow tracts was also established. They studied 14 patients, selected among 59 suffering from median nerve entrapment. Electrophysical confirmation of the diagnosis came from the EMG carried out on flexor carpi radialis and thenar muscles, which was normal on pronator teres. Conduction velocity was considered complementary, but not conclusive. Distal latency was normal, thus excluding carpal tunnel syndrome. Treatment confirmed the diagnosis: two patients underwent surgical exploration, 10 patients were treated with steroid injection into the pronator teres region, one patient with heat applications to the elbow, one patient refused treatment. All the treated patients improved.

F GIUFFRE, $V$ CONSOLI, $F$ VENTURA, $C$ MAZZARINO, AND M CAMPO (CATANIA) had studied serum levels of carcinoembryonic antigen (CEA) in brain tumours to see whether there was any correlation between histological tumour type and CEA levels. CEA serum levels were also monitored monthly after operation for six months to observe the eventual decrease as described in other tumours. Sixty patients aged between four and seventy-two years with brain tumours were studied before operation and followed up for six months after operation. CEA serum levels were determined by radioimmunological method using CIS Sorin Kits (Saluggia VC) with a tracer marked with ${ }^{125} \mathbf{I}$. All cases of meningioma showed CEA serum levels varying from 8 to $20 \mathrm{ng} / \mathrm{ml}$. Glial tumours also showed values ranging from 8 to 21 $\mathrm{ng} / \mathrm{ml}$. Metastatic tumours showed serum levels higher than those reported for primary tumours. Following neurosurgical treatment there was a steady reduction of CEA serum levels. Meningioma already showed a considerable decrease one month after operation. Almost similar was the situation for glioma, but the decrease was generally slower. In three cases of this group CEA serum levels began to increase a few months after operation, showing a clear correlation with recurrence. Metastatic tumours showed only a momentary decrease after operation. These results showed that a pre-operative diagnosis of brain tumour by this method is impossible, but there was evidence that careful monitoring of postoperative CEA serum levels could detect early occurrence of relapse, as observed in some of these cases.
M FONTANA, F CAROLI, CM CARAPELLA, AND A RICCIO (ROME) described a case of extra and intradural lumbar meningioma with a very long clinical history ( 29 years). It was possible to study retrospectively the progressive development of erosion of the second lumbar vertebral body. The patient was previously operated on $(25$ years ago) in another centre, for supposed L5/S1 left disc protrusion, without improvement of clinical findings. Recent myelography showed a complete block at L2 level with an unusual "migration" of the iodine contrast in the L2 vertebral body cavity. Operation showed an extensive meningioma from L1 to L3, densely adherent to the spinal nerve roots of the cauda equina and spreading into the L2 vertebral body. The presence of this tumour was ignored for 29 years and initially had been incorrectly diagnosed. The slow evolution of the clinical symptomatology apparently depended on progressive widening of the vertebral canal and on cavily formation in the L2 body; features unusual in spinal meningioma. They found it difficult to explain the pathogenesis of vertebral body cavitation; two different explanations being advanced, with respect to the myelographic picture. (1) "waterhammering mechanism"; the progressive L2 cavitation being due to continuous transmitted spinal fluid pulsation in an area of local thinning of the dura above the tumour, with dilatation of the subarachnoid space (2) The existence of a cyst at the cephalic pole of the tumour was they thought more probably responsible for L2 erosion: with the increasing intraspinal pressure the cyst wall ruptured thus opening into the subarachnoid space.

C DI ROCCO AND F VERLARDI (ROME) had studied 230 babies up to two years of age, admitted for a minor head injury. They were selected among 932 paediatric patients with head injury admitted to the Neurosurgical Clinic of the Catholic University of Rome in a seven year period. Most publications were specially concerned with severe head injuries, and generally, with all observed patients, from birth to adolescence. Knowledge appeared lacking of the final outcome of minor head injuries. In their opinion, this group of patients deserved isolated study according to their peculiar clinical and epidemiological characteristics. The age group represented a significant portion of cranial trauma in children. The inexperience and the negligence of the

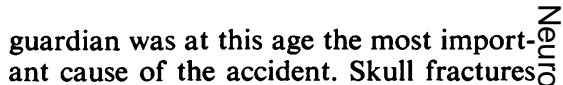
were not significantly correlated to signs of intracranial hypertension, nor to specific alterations in EEG pattern in 0 spite of their high incidence $(57 \%)$ in the group. The benign clinical evolution their $\stackrel{\rho}{工}$ group of patients could be referred to a hypothetical resistance to trauma of children at this age. However, there was:no difference in the final outcome when $\overline{\bar{s}}$ considering older patients who, at admission, presented clinical signs similar to those observed in their patients.

E TARTARINI, M BALDINI, M BOCCARDO, AND ّ̋ JP SICHEZ (GENOA AND PARIS) reported $\mathrm{a} \overrightarrow{0}$ series of nine supratentorial tuberculomas. They pointed out the difference in inci- $\vec{\omega}$ dence of tuberculoma between industriali- $D$ sed and underdeveloped countries, the greatest incidence being observed in? countries where tuberculosis is still an important illness. Their nine cases were typically European, supratentorial, pres-dominantly female and most in elderథy $v$ people. Among the neurological symptorigs and signs outstanding were those referabse

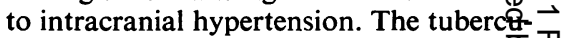
lin skin test and the chest $x$-ray films were $\mathbb{1}$ positive in only four cases before operation and only in these four pacients was preoperative chemotherapy instituted. The prevailing angiographic picture was th $\vec{\theta}$ of an avascular space-occupying lesion $\infty$ (seven out of nine cases). In six of their. cases, the tuberculoma appeared on the CT scan as a non-homogeneous, irregular contour, high attenuation value mass, $\frac{\partial}{\partial}$ surrounded by extensive oedema, in the remaining three the process presented $\frac{}{D}$ as an isodense area still surrounded by oedema. In six of their cases, block $\overrightarrow{\hat{\sigma}}$ removal of the tuberculoma was performed 3 while in the remaining three a piece-meal ablation of the mass was carried out. Allo patients underwent antituberculous treatment for four months following surgical treatment, and all patients survived. In? five cases no neurological sequelae were $\frac{\sigma}{3}$ present. The higher surgical mortality. reported by authors from under-developed $ᄋ$ countries might be explained by (1) the poor pre-operative general conditions, 을 (2) the presence of symptoms and signs of serious systemic tuberculous involvement, $\frac{D}{0}$ (3) the frequency of subtentorial localisation of the tuberculoma, (4) the high $\mathrm{O}$ percentage of cases observed in infancy and childhood. The rarity of intracranial $N$ tuberculomas in Europe and the absence 
of clear signs of systemic tubercular infection explain the frequency of preoperative misdiagnosis.

S BRIANI, P MENNONNA, M SOTTINI, G CAGNONI, $R$ GAGLIARDI, $L$ BENVENUTI, F GIARDINA, G GUIZZARDI, F AMMANATI, N TAVERNI, M COLAFRANCESCHI AND GL TADDEI (FLORENCE) presented a series of 273 cases of intracranial meningiomas operated on in Florence from 1961 to 1979. Among these cases only 26 recurrences $(9.5 \%)$ were established, either surgically or clinically. The incidence of recurrences in the different sites of origin was compared. In 172 cases histological review was carried out and correlation of tumour sites of recurrences and histological picture presented. This material was arranged according to the classification of Russel and Rubinstein, slightly modified by the consideration of the haemangiopericytic menigioma as a specific subtype. They concluded (1) recurrences of meningiomas have their highest incidence in parasagittal, basal and tentorial site where extirpation is sometimes necessarily incomplete, (2) histologically benign meningiomas do not recur when radically extirpated, which is nearly always possible on the cerebral convexity, (3) histologically malignant meningiomas and haemangiopericytic meningiomas recur in a higher percentage than other subtypes, but still with a highly different incidence according to the site of dural attachment and the consequent possibility or otherwise of a radical extirpation, (4) the combination of an unfavourable site with a possibly or truly malignant subtype has the greatest chance of a neoplastic recurrence.

B CIONI, M MEGLIO, P POLA, GF ROSSI, AND M SERRICCHIO (ROME) reported peripheral vascular changes induced by spinal cord stimulation. Spinal cord stimulation (SCS) via percutaneously insf:rted epidural electrodes was shown by Cook et al. (1976) and by Dooley and Kasprak (1976) to induce changes in peripheral circulation. The present work evaluated such an effect with both clinical and haemodynamic studies. Thirteen patients were examined. Ten of them had normal peripheral circulation: they were treated for chronic pain or neurogenic bladder. Three patients suffered for painful vascular disorders of the legs. Clinical observation included data from patients' reports and from pictures showing macroscopic tissue and vascular changes of the extremities. Instrumental examination included rheo- graphy, photoplethysmography, and in some cases, telethermography. Transcutaneous stimulation of the low-back and sham stimulation were studied as well. Both clinical and haemodynamic data showed that SCS induced a significant increase in peripheral blood flow. The effect was present both in subjects with normal and with impaired peripheral circulation. It appeared a few minutes after the beginning of stimulation and persisted some time after its cessation. The effect was apparent at the level of the microcirculation. In patients with peripheral vascular insufficiency circulation was remarkably increased following regular daily repetition of the SCS (longest follow up: 11 months). Some increase of blood flow on the periphery was shown also during transcutaneous stimulation. Such an increase was lower than the one observed during SCS and did not outlast it. Our preliminary data appeared encouraging in considering SCS as a useful treatment for patients with vascular diseases of the extremities.

NF KASSELl (IOWA) emphasised the dramatic improvement in the results of operation for ruptured aneurysm, but noted that overall management morbidity and mortality continued at unacceptable levels. Timing of surgery was an important factor in determining ultimate outcome, but the optimal interval remained controversial. He compared results in 20 patients admitted within three days of the most recent SAH assigned to "late" surgery with a similar group of 20 in whom "early" surgery was planned. In the late group surgery was deferred at least seven days after the haemorrhage until the patients were stable and had no vasospasm. Otherwise, surgery was deferred an additional seven days and the cycle repeated. In the early group surgery was performed within four days of the haemorrhage if there was no vasospasm on the pre-operative angiogram and no infarction by CT scan. admission and pre-operative neurological status was characterised as good-Hunts Grade I, II, IV, or poor-grades IA and III. Grade V patients were excluded from the study. Condition at follow up was classed as favourable outcome or dead. favourable outcome meant capable of independent, functional existence. The early and late groups were remarkably similar in terms of age, sex, neurological condition and aneurysm characteristics. Overall, in the late group $40 \%(8 / 20)$ had a favourable outcome and $45 \%(9 / 20)$ died, while in the early group $75 \%(15 / 20)$ had a favourable outcome and $15 \%(3 / 20)$ died. Operative complications occurred in the same frequency in both groups. In Phase VI of the co-operative aneurysm study, 249 patients were admitted within three days of subarachnoid haemorrhage and surgery was deferred at least 12 days. The results in that study were similar to those presented herein with $43 \%$ and $40 \%$ favourable outcome respectively. The results of early surgery reported by Suzuki and Sano were comparable to the $75 \%$ favourable outcome observed in this series. Furthermore, Hunt, Jane and Frazier all noted favourable results from early surgery, particularly in good condition patients. It appeared as a tentative conclusion that the overall favourable outcome in patients admitted within three days after last subarachnoid haemorrhage may be less than $45 \%$ if surgery is delayed one to two weeks in contrast to $75 \%$ if surgery is performed within the first four days. Were these differences to be widely confirmed, the potential impact could be enormous. It seems imperative that a definitive, conclusive answer to the timing of surgery controversy be reached $\stackrel{9}{\square}$

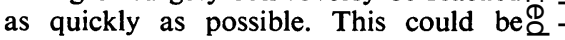
accomplished only through a large scale multi-centre, co-operative study.

MA GIOVANELli, $R$ VILlANI, SM GAINI, G TOMEI, AND A DUCATI (MILAN) had carried out 301 trans-sphenoidal operations at the Neurosurgical Department of the University of Milan in the past seven years. Two hundred and twenty six surgical procedures were prompted by presence of hypersecreting pituitary tumours (86 GH, 133 prolactin and seven ACTH secretors) and the remaining 75 by presence of non-secreting adenomas. A complete endocrine study was performed in all patients before and after surgery. Overall evaluation of results took into account a multiplicity of factors: sella changes, suprasellar expansion, surgical procedures (selective vs. radical ablation of the sella content). For acromegalics, surgery alone succeeded in normalising serum GH levels in $66 \%$ of patients. However, the rate of successes appeared to be largely different in two major groups of tumours. One group included all microadenomas, intra-pituitary adenomas larger than micro-adenomas with residual pituitary tissue and localised small invasive adenomas: in these tumours the rate of cure was altogether $85 \%$ (with some inequality between micro-ddenomas and 
the other tumour represented) while in the other group, that included all other adenomas, a failure rate of $50 \%$ or more, was to be expected. Postoperative radiotherapy (Co 60) further normalised serum GH levels in $30 \%$ of patients. Maximal efficacy of radiation therapy was obtained in the patients with postoperative $\mathrm{GH}$ levels below $30 \mathrm{ng} / \mathrm{ml}$. As for prolactin (PRL) secreting tumours, decrease to normal of serum PRL values was obtained in $76 \%$ of micro-adenomas. The rate of biological cure in the larger PRL secretors was altogether $20 \%$ with major differences between the intrasellar tumours $(36 \%$ of biological cures) and the adenomas with suprasellar extension of Guiot's grade I, II and III (12\% of biological cures). On the other hand, improvement of visual functions in the latter group of patients as in the nonsecreting tumours was obtained in $73 \%$ of cases. The efficacy of trans-sphenoidal procedures in obtaining a decompression of impaired optic pathways was confirmed. Nevertheless, postoperative radiotherapy has been shown to be imperative in order to maintain improved visual conditions and prevent later recurrences in this group of patients with suprasellar extension. Finally, four successes were obtained in the seven apparently delimited small ACTH secretors with Cushing's disease.

HE DIEMATH, J STROHECKER AND A FÜRST (SALZBURG) reviewed their experiences of stereotactic thermocontrolled radiofrequency (RF) heating of ganglion gasseri in trigeminal neuralgia. They pointed out that co-operative studies were always important in judging different methods applied to the same disease. Comparison could be difficult because of different patients (age, duration of history, general condition etc), and of different range of indications. In 116 patients they had operated on only those who had no or insufficient relief by drugs (carbamazepine). The type of operation was important since electrocoagulation did not necessarily mean "stereotactic thermocontrolled RF heating" (STRFH). Of 101 patients treated by STRFH as a first operation, 56 became painfree and 43 improved. There was no effect in three patients (follow up to 8 years). Fourteen of 25 patients who relapsed after STRFH became painfree after a repeated STRFH. Ten improved and the second procedure had no effect in one case. Once a Sjöqvist procedure and once a Frazier Operation was used to diminish recurring pain after STRFH. In 10 patients who had relapses after other previous operations seven became painless and three improved when STRFH was applied. It is considered that STRFH is a good procedure both as a first and second operation in repetition because (1) it is a minor operation and can be done on very old and weak patients without too great a risk, (2) the operative procedure is very safe because of the use of a stereotactic apparatus, $x$-ray control, control by electric stimulation and of temperature $\left(65^{\circ} \mathrm{C}, 30 \mathrm{~s}\right),(3)$ the amount of destruction can be limited due to intraoperative testing of sensibility and pain-conduction, (4) there is a very low rate of complications and side effects (none in our 89 last cases), (5) there are no technical problems in repeating this operation.

E PIERANGELI, VA LEVIN, LJ MARTON (BARI AND SAN FRANCISCO) studied the relationship of brain diffusion and capillary permeability putrescine $(\mathrm{Pu})$ to $\mathrm{CSF}$ levels in brain tumour patients. CSF Pu levels were extremely useful measures of tumour cell population in patients with medulloblastoma but not in patients with supratentorial malignant gliomas. Their study was designed to determine the diffusion co-efficient (D) for $\mathrm{Pu}$ in cat brain and the capillary permeability coefficient $(P)$ in rat brain to explain the failure of supratentorial gliomas to manifest a consistent increase in CSF $\mathrm{Pu}$ with progressive tumour growth. Ventriculocisternal (VC) and subarachnoid cisternal (SC) perfusions of cats were performed with an artificial CSF containing ${ }^{3} \mathrm{H}-\mathrm{Pu}$. Serial brain samples were taken from the caudate in the VC perfusions and from the cortex in the $\mathrm{SC}$ perfusions, and analysed for ${ }^{3} \mathrm{H}-\mathrm{Pu}$ specific radioactivity. Brain capillary permeability coefficients for ${ }^{3} \mathrm{H}-\mathrm{Pu}$ were studied in rat brain by injecting the isotope iv and taking serial blood samples and terminal cortex and subcortex. The samples were analysed for ${ }^{3} \mathrm{H}-\mathrm{Pu}$ specific radioactivity and the capillary peimeability cosfficients were computed. The $P$ was found to be elevated and the diffusion coefficient to be low. This implied that $\mathrm{Pu}$ crosses capillaries and enters cells reducing the amount of $\mathrm{Pu}$ which might ultimately reach the CSF by diffusion from the tumour. The data explained the fact that CSF levels of $\mathrm{Pu}$ correlate best in medulloblastomas since these tumours were generally locaied adjacent to the CSF pathways. CSF Pu levels correlated least in malignant supratentorial gliomas since these tumours were usually located within the brain hemispheres, and in order to increase CSF Pu levels the Pu must diffuse to the ventricles.

F LOEW, H JAKSCHE AND D NEUENFELDT (HOMBURG/SAAR) analysed 37 cases with non-communicating hydrocephalus, treated by the method of puncturing the third ventricule, and the results compared with published series in which other methods of third ventriculostomy had been used. The following conclusions were drawn: (1) in cases with non-communicating hydrocephalus caused by tumours of the posterior part of the third ventricle, the pineal region or near the aqueduct of Sylvius puncture third ventriculostomy was superior to all other methods, (2) in adults and elder children with hydrocephalus caused by nontumoural aqueduct stenosis, puncture third ventriculostomy gave good results in $80 \%$. Comparable figures of the results of Torkildsen procedure were about $70-80 \%$. Both methods were superior to extracranial shunting procedures like ventriculo-cardiac or 0 peritoneal shunts. Puncture third ventriculostomy was easier to perform and had $\frac{\rho}{\Phi}$ less complications than Torkildsen pro- $\varrho$ cedure, and therefore should be preferred. Repeated lumbar punctures during the $\Omega$ first postoperative days might be necessary to prevent secondary occlusion of the perforation of the third ventricular floor and while CSF resorption normalised. Extracranial shunting systems should only be implanted as a second treatment step when puncture third ventriculostomy failed. (3) non-communicating hydrocephalus caused by dysraphic malformations or following meningitis was not suitable for third ventriculostomy, and therefore should be treated primarily by shunting procedures, (4) the patency of the basal cisterns should be verified by CSF scintigraphy before performing third ventriculostomy. Cases with occluded basal cisterns should be excluded from this operation and treated by insertion of an extracranial shunting system.

A BOSELli, G SIGNORONI, R MASSEI (MILAN) had used depth EEG as the main diagnostic procedure in 160 cases of partial epilepsies treated since 1965 prior to CT. In all the cases electrocorticography (ECoG) was also performed, in order to obtain additional information on the epileptogenic area to be excised. Since CT was able to demonstrate cerebral lesions previously 
undetectable by standard radiological examinations, ECoG alone without prior depth EEG had recently been successfully employed in selected patients, when clinical, EEG and CT data point to the same circumscribed cortical area. The electrical identification of the epileptogenic area was felt to be mandatory even in cases with positive CT, as the simple removal of the macroscopic lesion may not prevent recurrence of the seizures. In their hands, surgery for epilepsy was performed under generalised anaesthesia, induced and maintained by Fentanyl iv, and nitrous oxide $(70 \%)$ and oxygen $(30 \%)$, with the patient curarised. At the time of ECoG, anaesthesia was lightened in order to have the patient awake with oxygen $(30 \%)$ and air, and curarised. The surgical flap was not limited to the area of the lesion, but was large enough to allow recording of the electrical activity from neighbouring regions. Eighteen electrodes were used, both on the exposed cortex and on the basal or mesial surfaces. Though the main aim of ECoG was the recording of spontaneous or induced seizures, prolonged recordings of cerebral activity during different functional states (anaesthesia, wakening) were also found helpful in localising the epileptogenic lesion. Prior to the scheduled surgery, blood serum levels of anticonvulsant drugs were monitored and maintained in a range at which spontaneous seizures occurred without clinical generalisation. Examples of ECoG were given in epileptogenic lesions successfully identified and removed.

GIULIO MORELLO AND GIOVANNI BROGGI (MILAN) had compared the long term results of open temporal rhizotomy and percutaneous thermorhizotomy, for trigeminal neuralgia. From 1955 to 1973 , 475 patients suffering from trigeminal neuralgia were operated upon at Instituto Neurologico of Milan by a modified Spiller-Frazier technique (SF). From 1974, this open procedure had been almost completely abandoned and the method of choice had been Sweet's differential radiofrequency retroganglionic thermorhizotomy (RFT), which has now been used for 615 patients. The authors compared a series of 409 patients who underwent the former procedure and were followed for at least two years and a series of 450 patients who had been treated by the latter method and had an identical follow up. Immediate failure occurred in
3 SF patients and in 32 RFT patients, all of them, except one who refused intervention, were re-operated on without delay and were pain free on discharge: therefore immediate success was achieved in $100 \%$ of RFT, and $99.8 \%$ of SF series. The mortality rate was $0.60 \%$ ( 3 cases) for SF and nill for RFT. Side effects were more frequent and severe with open surgery: hemiparesis appeared in seven patients after SF; it never appeared after RFT. Transient aphasia followed only seven procedures and was permanent in one case. Facial palsy or paresis never appeared after RFT while it was a transient sequelae for 16 patients $(3.6 \%)$ of SF series and permanent for three (0.97\%). Oculomotor deficit appeared after RFT in four cases and it was permanent in one, while it appeared in eight cases after SF, in one of which it was permanent. Motor function of trigeminal nerve was impaired in $17.5 \%$ of SF cases and in $4.7 \%$ of RFT cases. Painful anaesthesia or dysaesthesia was complained of in $1.7 \%$ of SF series and in $7.5 \%$ of RFT series. From the comparison between the two series they concluded that, even if RFT had some drawbacks, it gave better results than SF operation, with no mortality and lower risk of complications. It was recommended as the first choice surgical method while SF should be used as a last resort, in cases of repeated recurrences after RFT.

I PAPO, G CARUSELLI, A LUONGO, U PASQUINI, AND M SCARPELLI (ANCONA) surveyed their previous experience with the diagnosis and management of traumatic parenchymal brain lesions, and analysed the correlation between clinical CT scan and ICP data in 25 patients with traumatic intracerebral haematomas and/or brain lacerations. Clinically, the patients were classified in three groups, (1) deeply comatose patients ( 4 to 5 points on GCS.) -5 cases, (2) patients with intermediate disturbances of consciousness (6 to 10 points)-10 cases, (3) patients with minor impairment of consciousness (more than 10 points) -10 cases. Altogether 15 patients were operated upon. Surgery proved ineffective in the patients who were already deeply comatose in the first hours after injury, even though in some of them raised ICP was definitely reduced after operation. Conversely, the patients with well-limited lesions, moderate disorders of consciousness and persisting intracranial hypertension despite active therapy are excellent candidates for delayed surgery by limited procedures. It was $\underset{\mathbb{Q}}{Z}$ noticed as well that elevated ICP and/or severely increased elastance might last for a long time in patients with mild symptomatology. In some of such patients there was no close parallelism between clinical 0 and ICP time course. In patients with intermediate disturbances of conscious- $\frac{\vec{\partial}}{\bar{d}}$. ness and no tendency to improvement or $\stackrel{0}{\overrightarrow{3}}$ deterioration, ICP monitoring could be of $\leqslant$ practical use for making surgical decisions. However most cases diagnosed on CT scan $\stackrel{5}{+}$ had a benign course and recovered 0 uneventfully with conservative treatment. $\frac{\bar{\sigma}}{\bar{\sigma}}$ In these patients careful clinical observation was sufficient. On the whole, they con- $\frac{\mathbb{}}{\varnothing}$ cluded that surgical indications for the treatment of traumatic parenchymal brain $\omega$ lesions appeared to be rather limited.

R VILLANI, M GIOVANELLI, SM GAINI, TOMEI, $\vec{\omega}$ AND E MANGIAGALLI (MILAN) reviewed 123 cases of unilateral exophthalmos observed over the past 30 years, at the Neuro-? surgical Department of the University of $\vec{A}$ Milan, 85 of whom underwent surgical iv treatment. In these patients the exopti $\vec{V}$ thalmos could be accompanied by othe $v$ clinical signs, such as ophthalmoplegi@ 을 and/or impairment of visual functions. All patients underwent radiologict studies: plain films of orbit and skull weyc $\frac{D}{D}$ taken along with their tomograms, when needed. Angiography and venograph were helpful in assessing the diagnosis localisation in $70 \%$ of cases. CT scan wa available from the last five years; it allowed $\stackrel{\infty}{\rightarrow}$ clearcut evidence and sites of all orbital lesions and gave useful information about the nature of the pathology. Orbital surgery was performed throughout the $\bar{O}$ following routes: fronto-temporal-orbital

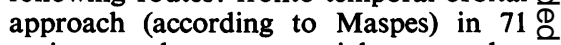
patients, other transcranial approaches $\overrightarrow{\vec{F}}$ in nine cases and orbital approach (Krönlein operation) in the remaining five cases. Neoplasms were found in 64 cases: 39 were benign and 24 showed histological features of malignancy. Most commonly observed were: cavernous haemangiomas, angiomas, osteomas, meningiomas, sarcomas, and congenital tumours of develop- 3 . mental origin. Non-tumoural masses were present in seven cases (abscesses, hydatid cysts etc) and pseudotumours in five. No expansive lesions could be found in nine 음 cases at surgery in the pre-CT scan period. $D$ Fifty-seven patients had follow up ranging from two to 20 years from surgical treatment. Current diagnostic possibilities, $\sigma$ surgical indications and techniques were $N$ discussed. 